\title{
2052 Interstudy reproducibility of the quantification of CMR myocardial late gadolinium enhancement in hypertrophic cardiomyopathy
}

\author{
Rory O'Hanlon*1, Agata Grasso ${ }^{1}$, Chiara Bucciarelli-Ducci' ${ }^{1}$ Rick Wage ${ }^{1}$, \\ Meghana Kulkarni ${ }^{1}$, Ali Alsafi ${ }^{2}$, Peter Gatehouse ${ }^{1}$, Michael Roughton ${ }^{2}$, \\ Dudley J Pennell ${ }^{1}$ and Sanjay K Prasad ${ }^{1}$
}

Address: ${ }^{1}$ Royal Brompton Hospital, London, UK and ${ }^{2}$ Imperial College, London, UK

* Corresponding author

from I th Annual SCMR Scientific Sessions

Los Angeles, CA, USA. I-3 February 2008

Published: 22 October 2008

Journal of Cardiovascular Magnetic Resonance 2008, I0(SuppI I):A32I doi:I0.I I86/I532-429X-I0-SI-A32I

This abstract is available from: http://jcmr-online.com/content/I0/SI/A32I

(c) 2008 O'Hanlon et al; licensee BioMed Central Ltd.

\begin{abstract}
Aim
Myocardial fibrosis is a common finding in patients with hypertrophic cardiomyopathy (HCM) and the amount of fibrosis is closely correlated with known risk factors for sudden cardiac death and adverse left ventricular remodeling. Quantification of fibrosis is challenging owing to the variable patterns and signal intensity of fibrotic regions, variable TI settings and surface coil responses. There are no published data on the interstudy reproducibility of late gadolinium quantification in HCM. This needs to be determined before the significance of longitudinal changes in fibrosis after pharmacologic or therapeutic interventions in defined patient cohorts can be assessed. We sought to assess the interstudy reproducibility of quantification of fibrosis in patients with HCM.
\end{abstract}

\section{Methods}

19 patients with HCM who underwent a clinical cardiovascular MR with gadolinium late enhancement (LGE) were invited back to have a repeat scan performed using the same protocol within a 4 week period from their index scan. The same operator performed both scans on a $1.5 \mathrm{~T}$ Siemens Avanto scanner with a 4 channel body array coil using the same scanning protocol. The gadolinium dose used was $0.1 \mathrm{mmol} / \mathrm{kg}$ and an inversion recovery segmented-FLASH imaging with TI adjusted to null normal myocardium to highlight regions of fibrosis was used (TR
$6.6 \mathrm{~ms}, 25^{\circ}, 23$ lines per cycle, $230 \mathrm{~Hz} /$ pixel) acquired 1.4 $\times 2.3 \times 8 \mathrm{~mm}$ voxels over typically $360 \times 270 \mathrm{~mm}$ FOV (adapted per patient)). A series of 10-12 short axis slices from base to apex were acquired for LGE analysis. Slice thickness was $8 \mathrm{~mm}$, and with a $2 \mathrm{~mm}$ gap between slices. An expert operator, blinded to patient clinical data, analyzed the scans and areas of fibrosis were manually traced and quantified in grams. Each of the two scans was analyzed at different time points to reduce bias. Owing to unavoidable slight misalignment between slices on different days, the total amount of fibrosis, in grams, was compared between scans as opposed the amount per slice.

\section{Results}

Coefficients of variation were calculated as the standard deviation of the differences between the two measurements divided by the mean value, and comparison of values was calculated using a Bland-Altman plot. Overall there was a good agreement between the 2 scans, and errors were less where amounts of fibrosis were smaller. The mean difference between the scans was -4.91 grams and the $95 \%$ limits of agreement were $(-25.39,15.56)$. The coefficient of variation for late gadolinium quantification in HCM was $19 \%$. 


\section{Discussion and conclusion}

This study demonstrates that manual quantification of fibrosis in HCM has reasonable interstudy reproducibility. The signal intensity of fibrotic regions detected by LGE is greater in infarcts than in HCM. For this reason it is challenging to accurately trace regions of fibrosis in HCM when there are large regions of differing signal intensities. Automated software analysis of fibrotic areas based on definitions of fibrosis as being 2 to 6 standard deviations from a user defined "normal" area of myocardium may be a more reproducible method of quantification between studies. Given the acceptable interstudy reproducibility, the quantification and follow up of fibrotic areas over time may be a reasonable endpoint to assess in therapeutic trials in HCM. 


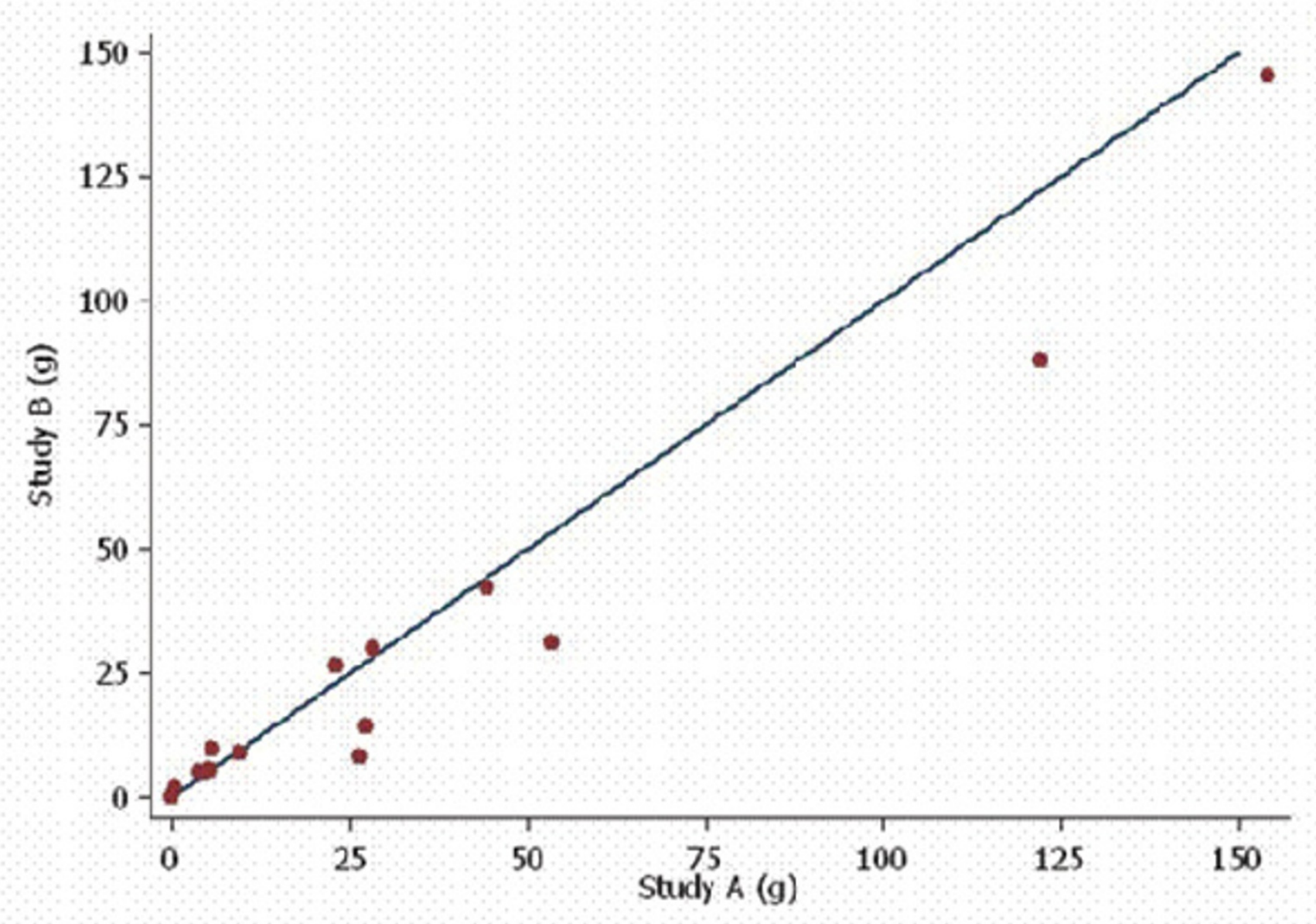

Figure I

Scattergram of the repeat LGE quantification in all 19 subjects with HCM. 


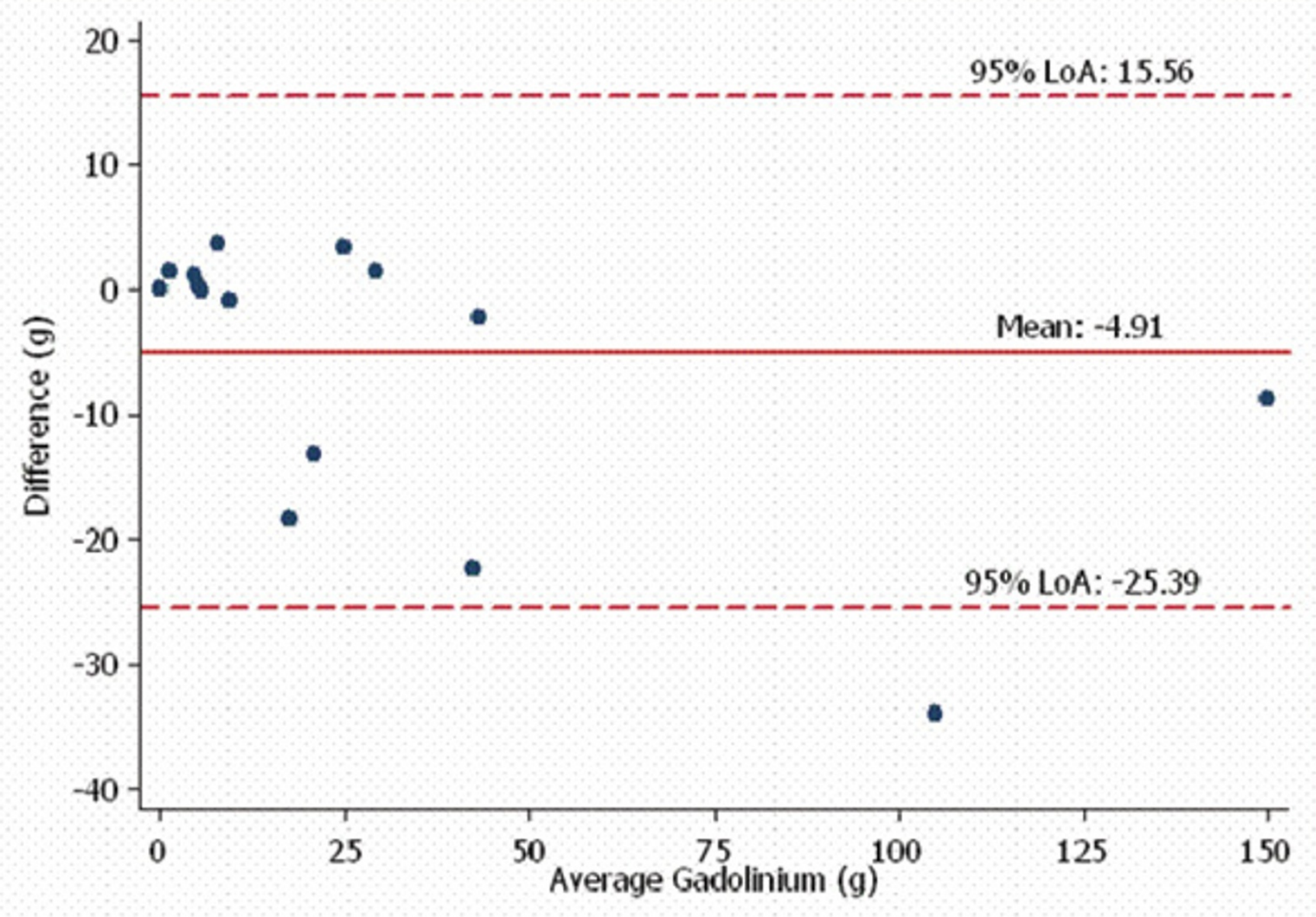

Figure 2

Bland Altman plot of HCM LGE interstudy reproducibility.

Publish with Bio Med Central and every scientist can read your work free of charge

"BioMed Central will be the most significant development for disseminating the results of biomedical research in our lifetime. "

Sir Paul Nurse, Cancer Research UK

Your research papers will be:

- available free of charge to the entire biomedical community

- peer reviewed and published immediately upon acceptance

- cited in PubMed and archived on PubMed Central

- yours - you keep the copyright
BioMedcentral 